\title{
Evolução do consumo no Brasil: da teoria à evidência empírica†
}

\author{
Fábio Augusto Reis Gomes*
}

RESUMO - Este artigo contrapõe a função consumo keynesiana e a abordagem intertemporal para discutir a evolução do consumo no Brasil. Segundo o modelo keynesiano, a renda disponível corrente é o único determinante do consumo, enquanto na abordagem intertemporal os consumidores otimizam sua escolha de consumo, sendo este dependente de vários fatores. A abordagem intertemporal pode ser vista como uma metodologia de construção de modelos, sendo flexível o bastante para incorporar modificações importantes, como a existência de restrição de crédito.

Palavras-chave: Consumo. Modelo keynesiano. Modelo intertemporal. Restrição de crédito. Brasil.

\section{INTRODUÇÃO}

O consumo agregado é uma das séries macroeconômicas de maior relevância, haja vista a sua expressiva participação no produto interno bruto (PIB), que é uma medida da soma de todos os bens e serviços finais (em valores monetários) produzidos no território de um país durante um determinado período. Por exemplo, de acordo com dados da versão 7.0 da Penn World Table, no ano de 2009 o consumo representava, em média, 71\% do PIB nos 190 países considerados. No caso do Brasil este percentual atingiu 75\%, enquanto nos Estados Unidos foi $79 \%{ }^{1}$.

Usando, ainda, os dados da versão 7.0 da Penn World Table referentes ao ano de 2009, a Tabela 1 apresenta o número e o percentual de países nos quais o consumo é superior à pelo menos 50\% do PIB. Por exemplo, dos 190 países considerados, 165 apresentaram consumo superior a $50 \%$ do PIB em 2009. Isso equivale a, aproximadamente, $87 \%$ dos países. Mesmo se elevarmos o percentual mínimo de consumo para 70\% do PIB, temos ainda 99 países que atendem a este critério, representando, assim, aproximadamente $52 \%$ da amostra.

\footnotetext{
† Agradeço a Afonso Henriques Borges Ferreira e Felipe Storch Damasceno pelos comentários, os isentando de quaisquer erros remanescentes.

* Doutor em Economia pela Fundação Getúlio Vargas. É professor da Fundação Instituto Capixaba de Pesquisas em Contabilidade, Economia e Finanças. Endereço eletrônico: fabiogomes@fucape.br.

1 Resultados obtidos considerando a variável kc, da versão 7.0 da Penn World Table, para 190 países que dispõem desta informação em 2009. A Penn World Table decompõe o PIB em três partes: consumo, investimento e gasto do governo. Foi utilizada a razão consumo-PIB. Isso explica a discrepância entre os resultados obtidos com base na Penn World Table e nos dados do IBGE apresentados abaixo.
} 
TABELA 1 - IMPORTÂNCIA DO CONSUMO NO PIB

\begin{tabular}{l|r|r}
\hline Percentual do consumo no PIB & Número de países & Percentual de países \\
\hline Maior do que $50 \%$ & 165 & $87 \%$ \\
Maior do que $60 \%$ & 146 & $77 \%$ \\
Maior do que $70 \%$ & 99 & $52 \%$ \\
Maior do que $80 \%$ & 55 & $29 \%$ \\
Maior do que $90 \%$ & 24 & $13 \%$ \\
\hline
\end{tabular}

FONTE: Elaboração própria.

NOTA: Dados extraídos da versão 7.0 da Penn World Table referentes a 190 países em 2009.

Usando dados anuais do Instituto Brasileiro de Geografia e Estatística (IBGE) referentes ao consumo final das famílias e ao PIB no Brasil, de 1947 a 2010, observamos que neste período a razão consumo-PIB esteve entre 54\% e 77\%, aproximadamente. Em 2010 tal razão foi de, aproximadamente, $61 \%$. Além de sua evidente importância como variável agregada, o consumo é um fator fundamental em qualquer análise de bem-estar da sociedade. Logo, a compreensão do seu processo de decisão e, por conseguinte, da sua evolução é fundamental.

Conforme discutido por Simonsen e Cysne (1995), Keynes (1936) propôs por introspecção, sem maiores fundamentos teóricos ou empíricos, a seguinte função consumo:

$$
C=C_{O}+c Y_{D}
$$

em que $C$ e $Y_{D}$ são, respectivamente, o consumo e a renda disponível, enquanto os parâmetros $C_{O}$ e $c$ são, respectivamente, o consumo autônomo e a propensão marginal à consumir. $\mathrm{O}$ consumo autônomo é positivo e independente da renda. Por sua vez, a propensão marginal a consumir pertence ao intervalo $(0,1)$, o que significa que aumentos da renda disponível se traduzem em aumentos do consumo proporcionalmente menores. Neste modelo, o consumo seria determinado exclusivamente pela renda disponível corrente e, por esta razão, este consumidor (keynesiano) é chamado de míope. Uma das consequências do modelo proposto por Keynes é que a propensão média a consumir, $C / Y_{D}$, é decrescente com relação à renda, ou seja, pessoas com renda mais alta poupariam uma maior proporção da renda que pessoas com renda mais baixa.

$\mathrm{Na}$ abordagem intertemporal, cujo pioneiro foi Irving Fisher, as famílias escolhem o consumo e a poupança no período corrente, levando em conta o futuro (ROMER, 2001). Portanto, o modelo intertemporal difere do modelo keynesiano em dois aspectos fundamentais. Primeiro, ao invés de seguir uma simples regra de consumir parte da renda disponível, o consumidor otimiza seu bem-estar ao escolher o consumo, levando em conta as informações disponíveis e a disponibilidade total de recursos. Como resultado, a escolha do quanto consumir 
depende, em geral, de outras variáveis, além da renda disponível corrente.

Em segundo lugar, a abordagem intertemporal pode ser vista como uma metodologia para construção de modelos de consumo com proposições testáveis (BROWNING; CROSSLEY, 2001). Em outras palavras, a ideia de que os consumidores maximizam seu bem-estar intertemporalmente, sujeitos à restrição orçamentária e ao conjunto de informações disponível, não gera proposições testáveis, a menos que hipóteses particulares sejam adotadas. De todo modo, mesmo os modelos intertemporais simplificados já levam a resultados bastante distintos daqueles implicados pela função consumo keynesiana. Por exemplo, os valores esperados da renda e do retorno (juros) futuros, além da riqueza, se tornam importantes determinantes do consumo.

$\mathrm{Na}$ próxima seção são apresentadas algumas versões simplificadas da abordagem intertemporal.

\section{MODELO INTERTEMPORAL DE CONSUMO}

Os precursores do modelo intertemporal, além de Irving Fisher, foram Franco Modigliani e Milton Friedman, que na década de 50 propuseram de forma independente a Teoria do Ciclo de Vida e a Teoria da Renda Permanente, respectivamente. Conforme os próprios títulos sugerem, Modigliani enfatizou que o horizonte de planejamento do consumidor é todo o seu tempo de vida, enquanto Friedman destacou o fato que a renda corrente não é o único determinante do consumo.

Modigliani desenvolveu a Teoria do Ciclo de Vida, basicamente, em dois artigos: Brumberg e Modigliani (1954) e Ando e Modigliani (1963). A contribuição de tal teoria está em considerar que a renda de um indivíduo sofre flutuações ao longo da sua vida; no entanto, com o objetivo de suavizar seu consumo, o indivíduo usa a poupança. Em geral, a renda tem formato em U invertido ao longo do tempo. Inicialmente a renda é baixa, mas ao longo do ciclo de vida eleva-se e, finalmente, volta a cair, especialmente em virtude da aposentadoria. Com o objetivo de suavizar o consumo, o indivíduo deveria acumular riqueza no período de renda alta para que o mesmo não se reduzisse drasticamente nos períodos de renda baixa. Por essa razão, a poupança dependeria da idade do indivíduo.

A Teoria da Renda Permanente, apresentada pela primeira vez em Friedman (1957), complementa a teoria de Modigliani, destacando que a renda sofre alterações, devido a sua composição, ao longo do ciclo de vida. Mais especificamente, Friedman considerou que a renda corrente é composta por dois elementos: a renda permanente, $Y^{P}$, e a renda transitória, $Y^{T}$. A renda permanente é a parte da renda que as pessoas esperam manter no futuro, ou seja, é quanto 
se pode consumir sem alterar a riqueza (CHAO, 2000). Já a renda transitória é a diferença entre a renda corrente e a renda permanente. Assim, se o consumidor consome a renda permanente, a poupança torna-se igual à renda transitória. Portanto, segundo a Teoria da Renda Permanente o consumo depende da renda permanente e os indivíduos usam a poupança e os empréstimos para suavizar o consumo em resposta a mudanças transitórias na renda corrente.

Flavin (1981) considerou a seguinte representação da renda permanente:

$$
Y_{t}^{P}=r\left[A_{t}+\sum_{i=0}^{\infty}\left(\frac{1}{1+r}\right)^{i+1} E_{t} Y_{l, t+i}\right]
$$

em que $Y_{t}^{P}, A_{t}$ e $Y_{l, t}$ são, respectivamente, a renda permanente, a riqueza e a renda do trabalho no período $t$, enquanto $r$ é a taxa de juros. O termo entre colchetes é a soma da riqueza real no período te do valor presente do fluxo esperado da renda do trabalho. Quando multiplicado por $r$, esse montante em valor presente gera um fluxo de recursos que, condicional a informação disponível em $t$, é constante e pode ser mantido durante todo o restante de horizonte de vida do indivíduo. O consumo seria, então, dependente da renda permanente, ao invés da renda corrente ${ }^{2}$.

Esses modelos intertemporais, ainda que muito simples, já trazem bastante informação sobre possíveis determinantes do consumo e da poupança, como a idade (ciclo de vida), a renda corrente e futura, os juros e a riqueza, bem como qualquer variável que afete o valor esperado da renda futura. É nesse sentido que a abordagem intertemporal se diferencia do caso keynesiano, no qual o consumo depende apenas da renda corrente.

$\mathrm{Na}$ próxima seção, discutimos a importância da renda corrente na determinação do consumo real per capita no Brasil. Conforme discutiremos, a renda corrente pode ser relevante por diversos motivos. Primeiro, a formulação keynesiana pode se mostrar apropriada. Segundo, a renda corrente pode conter informação sobre a renda permanente. Terceiro, em um ambiente no qual os consumidores não tem acesso a crédito, a suavização do consumo é bastante limitada e, por conseguinte, o consumo dependerá fortemente da renda corrente.

\section{EVIDÊNCIA EMPÍRICA}

Os modelos intertemporais expostos e o modelo keynesiano têm predições muito distintas. No modelo keynesiano, apenas a renda corrente importa e, por isso, o consumidor é, por vezes, chamado de míope. Nos outros modelos o consumidor é forward-looking, levando em

2 Alguns autores igualam o consumo a soma da renda permanente e de um componente transitório. No entanto, tal caso não será apresentado aqui. 
conta na sua tomada de decisão toda a informação disponível para estimar, inclusive, o valor esperado de variáveis futuras. Em princípio, uma maneira simples de avaliar estes modelos seria analisar a importância da renda corrente na determinação do consumo. O Gráfico 1 apresenta a dispersão do consumo contra o PIB, ambos em termos reais e per capita ${ }^{3}$. Percebe-se uma clara associação entre estas variáveis, conforme evidenciado pela reta de regressão ajustada pelo método de Mínimos Quadrados Ordinários (MQO). Nota-se, ainda, que a dispersão dos pontos em torno da reta de regressão é maior quando o PIB aumenta. Em outras palavras, a variabilidade do consumo parece depender da renda, havendo indícios de heterocedasticidade.

\section{GRÁFICO 1 - RELAÇÃO ENTRE O CONSUMO E O PIB}

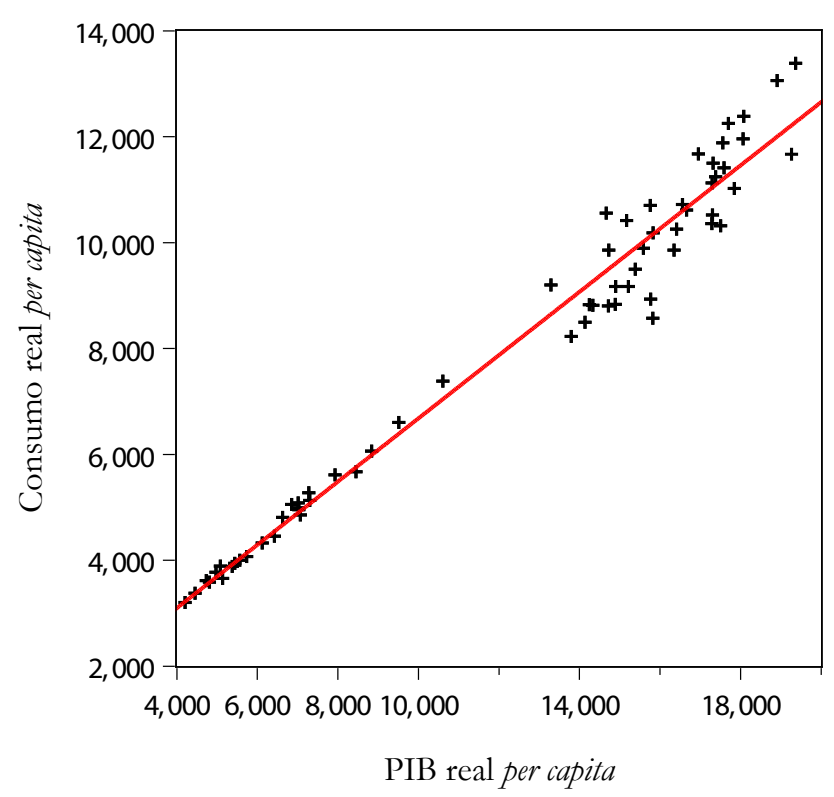

FONTE: Elaboração própria.

Na Tabela 2 apresentamos a estimação de um modelo de regressão simples no qual o consumo é a variável dependente e o PIB é a única variável independente ${ }^{4}$. Utilizamos dois métodos de estimação. O usual método de MQO e a Quantile Regression (QR) ${ }^{5}$. Enquanto o método de MQO estima o efeito médio da variável independente sobre a variável dependente, a QR estima o efeito da variável dependente sobre os quantis da distribuição condicional da variável dependente.

A QR é especialmente interessante no caso do consumo, pois, quando há heteroce-

3 Em todas as análises empíricas feitas nesta seção são utilizados os dados de consumo final das famílias e PIB do Brasil de 1947 a 2010, calculados pelo IBGE. Os dados foram deflacionados pelo IGP-DI e divididos pela população residente, de modo a obtermos dados em termos reais e per capita. Todos estes dados foram extraídos do IPEADATA.

4 Após a estimação deste modelo de regressão simples via método de MQO, a análise dos resíduos indica que o mesmo é integrado de ordem zero, de modo que não incorremos no problema de regressão espúria.

5 Ver Koenker e Basset (1978). 
dasticidade, o efeito médio não é um bom estimador do que acontece ao longo dos quantis da distribuição da variável dependente. Estimamos, então, o impacto do PIB sobre os seguintes percentis da distribuição do consumo: 0,$10 ; 0,25 ; 0,50 ; 0,75$ e 0,90. Todas as estimativas são significativamente diferentes de zero, quando são considerados os níveis de significância habituais, $1 \%, 5 \%$ e $10 \%$. A estimativa de MQO sugere que o coeficiente do PIB real per capita é igual a 0,60 , indicando que a cada aumento de uma unidade monetária desta variável, o consumo aumenta, em média, 0,60 unidades monetárias. Os resultados provenientes do método de QR indicam que nos percentis mais altos da distribuição condicional do consumo o impacto do PIB é maior, havendo um efeito assimétrico ao longo de tal distribuição.

TABELA 2 - ESTIMAÇÕES PARA A RELAÇÃO ENTRE O CONSUMO E O PIB

\begin{tabular}{lrrrrrr}
\hline \multicolumn{7}{c}{ Variável dependente: Consumo real per capita } \\
Período: 1947 a 2010
\end{tabular}

FONTE: Elaboração própria.

NOTA: MQO e QR referem-se, respectivamente, ao estimador de Mínimos Quadrados Ordinários e a Quantile Regression. Entre parênteses estão os erros-padrão robustos.

Estes resultados sugerem que a renda corrente é um determinante importante do consumo, especialmente se considerarmos o $\mathrm{R}^{2}=0,97$ obtido na estimação por MQO. Daí, poderíamos concluir que o modelo keynesiano é extremamente adequado. No entanto, esta interpretação apresenta dois problemas. Primeiro, a renda corrente pode prever bem a renda futura e, portanto, isso explicaria o êxito do modelo acima ainda que a função consumo keynesiana não seja adequada. Neste caso, a renda corrente serviria como uma boa proxy para a renda permanente. Segundo, em um ambiente com pouco acesso ao crédito, os consumidores podem não conseguir suavizar o consumo, estando fadados a utilizar, basicamente, a renda corrente. Nesta perspectiva, os consumidores não seriam míopes, mas o modelo keynesiano seria empiricamente bem-sucedido por gerar uma proposição testável similar àquela gerada pela restrição de crédito.

Vamos examinar o primeiro caso. Suponha, por simplicidade, que a renda do trabalho segue um processo autorregressivo de ordem 1 , tal que $Y_{l, t}=\mu+\Phi Y_{l, t-1}+\xi_{t}$, em que $\mu$ e $\Phi$ são constantes e $\xi_{t}$ é o termo de erro. Então, a renda permanente, expressa na Equação (2), torna-se: 


$$
Y_{t}^{P}=\tilde{\mu}+r A_{t}+\frac{r}{1+r-\Phi} Y_{l, t}
$$

em que $\tilde{\mu}$ é uma constante que depende de $\mu, \Phi$ e $r$. Portanto, se a renda corrente é usada para prever a renda futura, a dependência do consumo em relação à renda corrente não significa que os modelos intertemporais sejam desnecessários. De fato, se a renda corrente é correlacionada com a riqueza, ela pode se tornar uma proxy para tal variável também e, por conseguinte, para a renda permanente como um todo. Tudo isso nos levaria a superestimar, erroneamente, a importância da renda corrente. Note que um teste mais adequado poderia ser realizado caso dispuséssemos de informação sobre a riqueza, pois a relevância de tal variável seria uma evidência contra a função consumo keynesiana.

Considere o caso no qual a renda do trabalho dos indivíduos tem formato de U invertido ao longo da vida, conforme o Gráfico 2. Para que o consumo seja suave, conforme a linha horizontal, é necessário que o consumidor tome emprestado na juventude, pois o consumo (suavizado) seria superior a renda em tal período. $\mathrm{Na}$ fase adulta ele pouparia para pagar sua dívida e para financiar a manutenção do consumo na velhice, haja vista a queda da renda em tal período. Logo, a suavização do consumo pressupõe a existência de um mercado de crédito no qual a renda do trabalho futura é um colateral aceito para empréstimos.

\section{GRÁFICO 2 - SUAVIZAÇÃO DO CONSUMO}

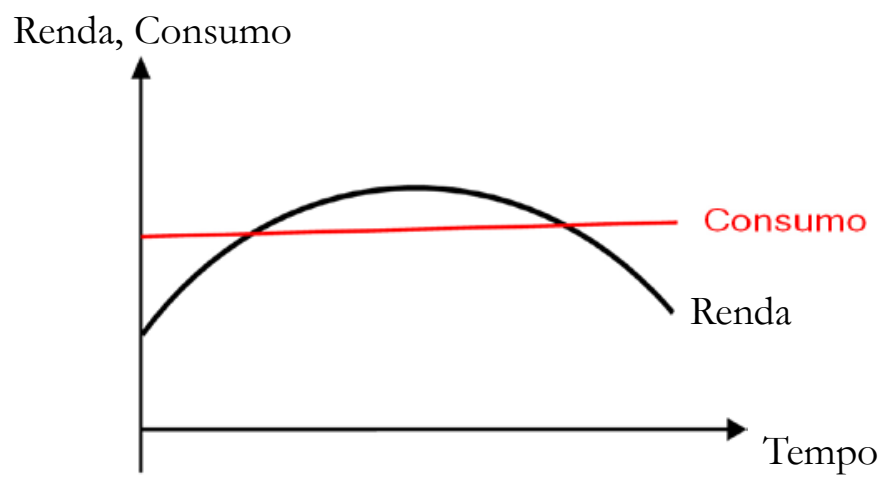

FONTE: Elaboração própria.

Estamos utilizando uma versão bastante simplificada da abordagem intertemporal, no entanto, as versões mais usuais desta abordagem também supõem que existe um mercado de crédito desenvolvido ${ }^{6}$. Nesta perspectiva, as versões mais usuais da abordagem intertemporal e da teoria keynesiana constituem dois extremos. No primeiro caso, é fundamental a existência

6 Ver, por exemplo, Hall (1978), Hall (1988), Yogo (2004) e Brady (2008). 
de crédito, enquanto no segundo o consumo é financiado apenas pela renda corrente, não importando o crédito. Na prática, uma situação intermediária pode ser mais profícua. Os consumidores têm algum acesso ao crédito e, embora a renda corrente seja muito importante, outros fatores também devem afetar o consumo.

Como o arcabouço intertemporal é, de fato, uma metodologia de construção de modelos, a restrição de crédito pode ser incorporada à estrutura do modelo. Alguns exemplos incluem Zeldes (1989), Ludvingson (2000) e Scott (2010). Já o modelo keynesiano não possui tal flexibilidade, não incorporando, portanto, outros elementos adicionais que afetam o consumo.

Shea (1995) argumenta que consumidores míopes têm seu consumo totalmente dependente da renda corrente, enquanto consumidores restritos ao crédito estão impedidos de tomar emprestado, mas podem acumular poupança. Por esta razão, quando a renda aumenta o consumo deveria responder a tal aumento; porém, quando a renda cai, o consumidor pode usar sua poupança evitando, inclusive, uma queda no consumo. O Gráfico 3 apresenta a dispersão da taxa de crescimento do consumo real per capita e do PIB real per capita, entre 1947 e 2010.

Delimitamos com a linha horizontal e a linha vertical quatro quadrantes. Quando a taxa de crescimento da renda e do consumo tem o mesmo sinal, isso é compatível com comportamento míope, restrição de crédito e suavização de consumo. Por essa razão, dois quadrantes são inconclusivos e representam, portanto, uma situação de indefinição.

\section{GRÁFICO 3 - RELAÇÃO ENTRE O CRESCIMENTO DO PIB E DO CONSUMO}

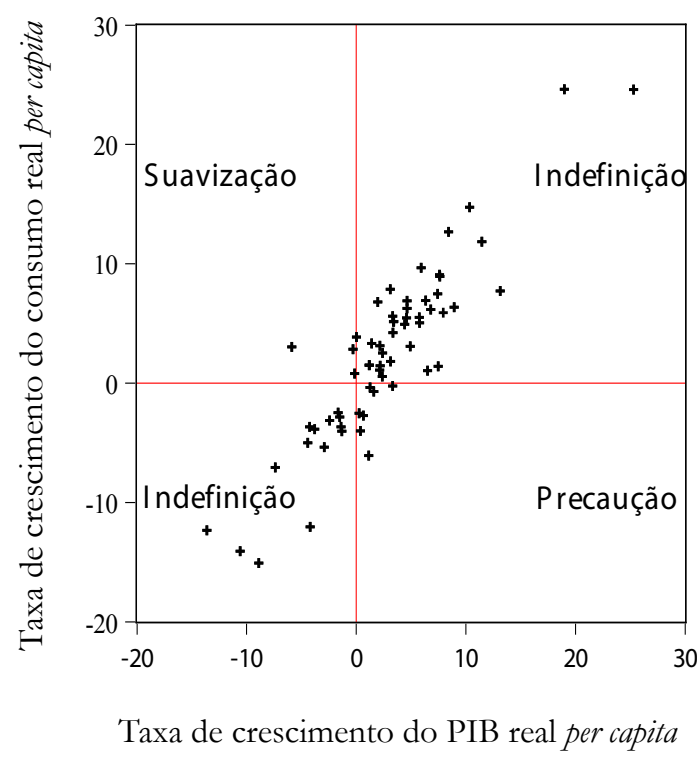

FONTE: Elaboração própria.

Para ser preciso, é importante dizer que, quando após uma queda da renda o consumo cai menos do que a renda, temos uma evidência de suavização de consumo, pois se o indivíduo 
fosse míope a variação do consumo deveria ser idêntica a da renda. Porém, como é utilizada uma medida imprecisa dos recursos do consumidor, o PIB, vamos nos ater aos casos, digamos, extremos de suavização: situações nas quais, a despeito da queda do PIB, o consumo aumenta. No quadrante intitulado suavização isso ocorre. Por fim, no quadrante intitulado precaução a situação oposta ocorre, pois enquanto o PIB subiu, as famílias decidiram reduzir o consumo. Estes dois fenômenos são detalhados na Tabela 3. Quanto à suavização, chama atenção a diferença entre a taxa de crescimento do consumo e da renda em 1990 (Plano Collor). No quesito precaução observamos, por exemplo, uma retração do consumo na crise de 2008 , embora o PIB tenha crescido. Neste cenário de crise (elevado risco) os consumidores preferiram ser cautelosos, reduzindo o consumo, mesmo com o PIB crescendo.

TABELA 3 - ANÁLISE DA RELAÇÃO ENTE CRESCIMENTO DO CONSUMO E PIB

\begin{tabular}{l|r|r|r}
\hline \multirow{2}{*}{ Comportamento do consumo } & \multirow{2}{*}{ Ano } & \multicolumn{2}{c}{ Taxa de crescimento real per capita } \\
\cline { 3 - 4 } & & Consumo & \multicolumn{1}{c}{ PIB } \\
\hline \multirow{3}{*}{ Suavização } & 1964 & $0.79 \%$ & $-0.16 \%$ \\
& 1967 & $2.85 \%$ & $-0.29 \%$ \\
& 1990 & $3.02 \%$ & $-5.89 \%$ \\
\hline \multirow{3}{*}{ Precaução } & 1950 & $-0.71 \%$ & $1.60 \%$ \\
& 1953 & $-6.08 \%$ & $1.14 \%$ \\
& 1963 & $-2.53 \%$ & $0.27 \%$ \\
& 1965 & $-2.72 \%$ & $0.66 \%$ \\
& 1989 & $-4.02 \%$ & $0.38 \%$ \\
& 2004 & $-0.26 \%$ & $3.33 \%$ \\
& 2008 & $-0.38 \%$ & $1.25 \%$ \\
\hline
\end{tabular}

FONTE: Elaboração própria.

Em síntese, há evidência de que, pelo menos algumas vezes, os consumidores usaram a poupança acumulada atenuando o efeito da queda do PIB sobre o consumo. Por vezes, também, o consumidor escolheu poupar mais, mesmo com o PIB aumentando. Tudo isso indica que o consumidor não é míope. O desafio torna-se, então, encontrar um modelo com a dose certa de restrição ao crédito, havendo alguma flexibilidade para a escolha dos consumidores.

Os resultados obtidos são em alguma medida sensíveis a certas limitações. Por exemplo, não dispomos de uma série longa de consumo de não duráveis. Portanto, a série utilizada é composta por bens duráveis e não duráveis, o que não é adequado ou, pelo menos, seria interessante analisar tais tipos de bens separadamente. Usamos o IGP-DI como deflator ao invés do IPCA, que é o índice considerado no sistema de metas de inflação. A razão é simples: não dispomos do IPCA desde 1947, início da amostra. Naturalmente, os resultados podem sofrer alguma alteração se outro índice de inflação for usado. De todo modo, os resultados obtidos são similares àqueles obtidos na literatura nacional. Por exemplo, Gomes (2010) construiu uma figura similar ao Gráfico 3, obtendo resultados parecidos. 


\section{CONCLUSÕES}

A dependência do consumo em relação à renda corrente é compatível com diversas abordagens. No entanto, os pontos nos quadrantes suavização e precaução evidenciam que os consumidores não são míopes. O processo decisório dos consumidores é mais sofisticado do que o estabelecido pela função consumo keynesiana.

O Brasil passa por um momento relativamente novo no qual há um crescimento do crédito. Certamente, os consumidores ainda estão restritos ao crédito, porém cada vez menos. Daí a pergunta: quais serão os efeitos do aumento do crédito? Teoricamente, quanto maior a disponibilidade de crédito maior a importância da renda permanente em detrimento da renda corrente. Deste modo, políticas econômicas que afetam a renda permanente ao invés da renda corrente, a partir de algum momento, terão mais impacto sobre o comportamento do consumidor. Obviamente, saber quando exatamente isso ocorrerá é muito difícil. De fato, se a renda corrente contiver bastante informação sobre a renda permanente, esta distinção fica muito entenebrecida.

Como um consumidor que quase nunca teve acesso ao crédito está se comportando neste novo cenário? Qualquer pessoa que contrai uma dívida tem alguma chance de não conseguir pagá-la. Isso ocorre por uma razão simples: imprevistos ocorrem. Se o consumidor faz um empréstimo e perde o emprego, sua capacidade de honrar sua dívida fica muito comprometida. Quando ocorre um choque negativo de qualquer natureza, a probabilidade de calote aumenta. Ora, se mais pessoas estão tomando emprestado, mais pessoas estão sujeitas a esse risco e é possível que a inadimplência aumente.

A recente decisão do Banco Central de aumentar o pagamento mínimo do cartão de crédito foi bastante impopular, o que significa que muitas pessoas escolhem se endividar no cartão de crédito. $\mathrm{O}$ ato de comprar no cartão de crédito não significa fazer uma dívida. Mas, ao não pagar a fatura total do cartão de crédito, o consumidor contrai uma dívida e, portanto, paga os juros da mesma. Os juros do cartão de crédito estão em torno de $10 \%$ ao mês.

É importante dizer que o cartão de crédito não é, necessariamente, o instrumento de compra das classes mais pobres. Essas, por vezes, usam crediário ou cartões de crédito de lojas. Este cartão é aceito apenas na loja que o emite, sendo, na prática, muito similar a um crediário, cuja taxa está em torno de $6 \%$ ao mês $^{7}$. É interessante notar que algumas lojas parecem cumprir dois papéis: vendem produtos e emprestam crédito, haja vista o extenso parcelamento das compras e o fato de que em alguns casos a compra à vista não tem desconto. Portanto, tais lojas

7 Fonte para estas taxas de juros: <http://www.infomoney.com.br/indicadores $>$. Valores referentes a julho de 2011. 
têm interesse em financiar seu consumidor.

O crédito não é ruim. No entanto, é preciso se adaptar a esta nova realidade. Ao tomar emprestado, o consumidor deve considerar as diferentes opções de crédito, escolhendo aquela de menor custo.

\section{REFERÊNCIAS}

ANDO, A.; MODIGLIANI, F. The "life cycle" hypothesis of saving: aggregate implications and tests. The American Economic Review, v. 53, n. 1, p. 55-84, 1963.

ATEN, B.; HESTON, A.; SUMMERS, R. Penn world table version 7.0. Center for International Comparisons of Production, Income and Prices at the University of Pennsylvania. 2011.

BASSET, G.; KOENKER, R. Regression quantiles. Econometrica, v. 46, n. 1, p. 33-50, 1978. BRADY, R. Structural breaks and consumer credit: is consumption smoothing finally a reality? Journal of Macroeconomics, v. 30, n. 3, p. 1246-1268, 2008.

BROWNING, M.; CROSSLEY, T. The life-cycle model of consumption and saving. Journal of Economic Perspectives, v. 15, n. 3, p. 3-22, 2001.

BRUMBERG, R.; MODIGLIANI, F. Utility analysis and the consumption function: an interpretation of cross-section data. In: KURIHARA, K. (Ed.). Post-keynesian economic. New Brunswick: Rutgers University Press, 1954, p. 388-436.

CAMPBELL, J.; MANKIW, G. Consumption, income and interest rates: reinterpreting the time series evidence, In: OLIVIER, J.; FISCHER, S. (Org.). National Bureau of Economic Research macroeconomics annual. Cambridge: MIT Press, 1989, p. 185-216.

CHAO, H. Milton Friedman and the emergence of the permanent income hypothesis. Tinbergen Institute Discussion Paper, n. 53, nov. 2000.

FLAVIN, M. The adjustment of consumption to changing expectations about future income. Journal of Political Economy, v. 89, n. 5, p. 974-1009, 1981.

FRIEDMAN, M. A theory of the consumption function. Princeton: Princeton University Press, 1957.

GOMES, F. Consumo no Brasil: comportamento otimizador, restrição de crédito ou miopia? Revista Brasileira de Economia, v. 64, n. 3, p. 261-275, 2010.

HALL, R. Stochastic implications of the life cycle-permanent income hypothesis: theory and evidence. Journal of Political Economy, v. 86, n. 6, p. 971-987, 1978.

HALL, R. Intertemporal Substitution in Consumption. Journal of Political Economy, v. 96, n. 2, p. 339-57, 1988.

KEYNES, J. The general theory of employment, interest and money. London: Macmilian, 1936

LUDVIGSON, S. Consumption and credit: a model of time-varying liquidity constraints. The Review of Economics and Statistics, v. 81, n. 3, p. 434-447, 1999.

ROMER, D. Advanced macroeconomics. 2. ed. Boston: McGraw-Hill: Irwin, 2001.

SIMONSEN, M.; CYSNE, R. Macroeconomia. 2. ed. Rio de Janeiro: FGV: Atlas, 1995. 
SCOTT, A. Optimal consumption under imperfect capital markets. Economics Letters, v. 66, n. 1, p. 65-70, 2000.

ZELDES, S. Consumption and liquidity constraints: an empirical investigation. Journal of Political Economy, v. 97, n. 2, p. 305-46, 1989.

YOGO, M. Estimating the elasticity of intertemporal substitution when instruments are weak. Review of Economics and Statistics, v. 86, n. 3, p. 797-810, 2004. 